REVISTA IBEROAMERICANA DE EDUCACIÓN A DISTANCIA

Vol. $5 \cdot \mathrm{N} .^{\circ} 2$

Diciembre, 2002

\title{
Resistencias, cambio y buenas prácticas en la nueva educación a distancia
}

\author{
(Resistances, change and good practices in the new distance education) \\ LORENZO GARCÍA ARETIO \\ Cátedra UNESCO de Educación a Distancia, UNED-España \\ www.uned.es/cued
}

SUMARIO: Tras defender la necesidad de bases teóricas sólidas que justifiquen las buenas prácticas en educación a distancia, se describen las principales resistencias al cambio desde una pedagogía basada en la relación presencial a otra apoyada en la interacción mediada. Ahora bien, a lo largo del trabajo, se mantiene que los cambios a que pueden obligar la incorporación de las tecnologías a los ámbitos educativos, no afectan sustancialmente a los principios pedagógicos fundamentales. Finalmente, se argumenta sobre cuáles deben ser las acciones consideradas prioritarias para garantizar buenas prácticas en educación a distancia: investigación, formación, planificación, evaluación y establecimiento de redes.

Educación a distancia - buenas prácticas - principios pedagógicos - cambios

ABSTRACT: After defending the need of theoretical solid bases that justify and encourage the good practices in Distance Education, it isdescribed in the article the major resistances to the change from a pedagogy based on the face-to-face relation to other one that leans on themedia interaction. Nevertheless, it is stated in the paper that the changes that may be forced by the incorporation of the technologies to the educational areas do not affect substantially the pedagogic fundamental principles. Finally, theactions considered to be the most important to guarantee good practices in distance education are discussed: research, training, planning, evaluation and establishment of nets.

Distance education - good practices - pedagogic principles - changes 


\section{INTRODUCCIÓN}

Nadie con un grado mínimo de sensatez académica pone en duda la necesidad de contar con fundamentos teóricos que alimenten, justifiquen, guíen, provean de significados y faciliten futuros desarrollos para las realizaciones prácticas. Son las ideas, surgidas del mundo de las teorías las que marcan los núcleos fundamentales, revelan nuevas formas de conocer y sugieren alternativas de acción. Las realizaciones prácticas eficaces y de calidad, y la educación debe ser incuestionablemente una de ellas, deben basarse en postulados teóricos sólidos, coherentes y rigurosos. Cuando un desarrollo educativo completo se muestra como eficaz, una de dos, o está fuertemente fundamentado en algunas ideas teóricas o es fruto de la fortuna (García Aretio, 2001). Pero incluso en este último caso, podríamos concluir que el citado desarrollo se ajusta a un determinado modelo teórico o a más de uno, aún cuando el protagonista del mismo lo ignore. La teoría es fundamental para entender y transmitir las propuestas, métodos y objetivos de cualquier realización práctica (Garrison, 2000). Y en nuestro caso el punto de mira se dirige a la educación a distancia.

Siempre hemos defendido que para el desarrollo de prácticas adecuadas, se precisan bases teóricas sólidas. Deberíamos esforzarnos para que esas prácticas quedasen resueltamente condicionadas por coherentes y rigurosos estudios teóricos que nos permitiesen sistematizar las leyes, ideas, principios y normas, con objeto de describir, explicar, comprender y predecir el fenómeno educativo en la modalidad a distancia y regular la intervención pedagógica en este ámbito (García Aretio, 1989 y 1994).

Estas construcciones teóricas han de desarrollarse, desde la propia práctica y, a la vez, para mejorar ésta. Y sus agentes habrán de ser, en nuestro caso referidos a la educación a distancia, docentes e investigadores convencidos de sus bondades y capacitados para esa tarea. Por otra parte, la aplicación práctica de los supuestos y desarrollos teóricos relativos a la educación a distancia exigirá, al igual que en otras modalidades, cuadros directivos, docentes y administrativos debidamente cualificados.

Esas mismas propuestas teóricas, para ser debidamente aplicadas a desarrollos prácticos, requerirán de planificadores y diseñadores de las instituciones y programas a distancia que ofrezcan proyectos sólidos soportados en rigurosos análisis, experiencias previas y bases teóricas. Y, ¿cómo olvidar para el logro de buenas prácticas, la evaluación de la calidad de esas instituciones y programas? Ya se sabe que el ciclo completo supone: planificación, desarrollo, evaluación o control y propuestas para la mejora y consecución de buenas prácticas.

Ayuda a todo lo que llevamos proponiendo, el establecimiento de redes institucionales y, no lo olvidemos, de intercambio, debate y trabajo colaborativo entre miembros individuales que se desempeñan en instituciones y contextos geográficos y sociales diferentes. 
Resistencias, cambio y buenas prácticas en la nueva educación a distancia

Las tecnologías que irrumpen en el ámbito de la educación están exigiendo cambios en las formas de pensar y hacer la misma. Nosotros participamos de las bondades de la innovación y el cambio, pero como son muchos los que, con la incorporación de las tecnologías, hablan de cambios radicales en la educación, nuevos y prometedores paradigmas educativos, revolución pedagógica, etc., nos preguntamos ¿en realidad es tanto lo que cambia en la educación a distancia con la aplicación, incorporación o, mejor, integración de las nuevas tecnologías a los procesos formativos?, ¿cambian las bases o sólo los procedimientos? Las buenas prácticas nos exigen ser conscientes de la dirección, grado e intensidad de los cambios que se postulan.

A pesar de todo lo anteriormente expuesto, de poco valdrían las bases teóricas conformadas tras rigurosos estudios e investigaciones, la capacitación de los recursos que habrían de implementar el hipotético programa, la detallada planificación, la evaluación, etc., si se ignoran las posibles resistencias a la innovación educativa, al cambio. Y, además, ciertamente poco cambia o puede cambiar si no se cuenta con los necesarios apoyos políticos, sociales e institucionales.

En fin, lo dicho hasta aquí lo podríamos resumir señalando que en las siguientes propuestas podrían estar las bases para las buenas prácticas en una educación a distancia de hoy, amén de reiterar que como sustrato de estas bases habremos de considerar siempre los aportes teóricos al campo:

D Eliminación o reducción de las resistencias al cambio.

Deconocimiento y consciencia de lo que realmente cambia o puede cambiar.

Donsciencia de las dificultades que supone la incorporación de las tecnologías.

D Investigación sobre las entradas, procesos y resultados.

Dapacitación de cuadros directivos, gestores y, sobre todo, docentes.

Delevancia de la planificación para asegurar el éxito.

D Implantación de procesos serios de evaluación institucional y de programas que aseguren la calidad.

D Participación en redes internacionales de carácter institucional o personal.

D Afán por la búsqueda de apoyos políticos, sociales e institucionales.

\section{DE LAS RESISTENCIAS E INMOVILISMO..., AL CAMBIO «PORQUE TOCA»}

Introducir innovaciones en las instituciones y programas de educación a distancia ya establecidos y con estilos más clásicos o en las instituciones y progra- 
mas presenciales que deciden incorporar esta modalidad, suele traer consigo dificultades que pueden provenir de la acción en contra de individuos o grupos para que esos cambios se lleven a cabo, o de los problemas u obstáculos insalvables que puede encontrar en su camino tal intención innovadora, que aunque no actúen operativamente en contra, han de ser superados, porque están ahí.

Para nosotros la innovación, si la deseamos aplicar a instituciones y programas a distancia, la entenderíamos como la acción deliberada de introducir algún cambio que transforme la estructura, los componentes o procesos de la citada institución o programa o de la propia práctica docente, con el fin de mejorarlos o de ofrecer otras alternativas educativas igualmente eficaces (García Aretio, 1994).

Sabemos que existen una serie de factores, fuentes o bases de las resistencias a la innovación en educación (García Aretio, 1992 y 1994). Las resistencias al cambio son de siempre por lo que no debemos alarmarnos con las existentes hoy ante la incorporación de las tecnologías a la educación. Ya existieron, incluso, ante la incursión y expansión de la escritura, como muestra lo que Platón, en el Fedro le hacía decir a su Sócrates: «es (la escritura) inhumana al pretender establecer fuera del pensamiento lo que en realidad sólo puede existir dentro de él... La escritura destruye la memoria... debilita el pensamiento». Es también lo que hoy señalan algunos respecto al uso de las calculadoras por parte de los niños. La propia imprenta suscitó muchos recelos al suponerse que podía destruir la memoria y debilitar el pensamiento (Ong, 1987)

Los factores, fuentes o bases de la resistencia al cambio educativo en general, y a la introducción de las tecnologías en particular, son o pueden ser muchos. Entro otros, podríamos apuntar los siguientes:

D Oposición activa de individuos o grupos, tanto externos como internos a la institución.

D Valores, normas y estructuras del sistema educativo generalmente muy arraigados.

Desconocimiento o ignorancia sobre las posibilidades de los cambios que se proponen.

D Temor a ser superados por los propios alumnos o a tener que reconsiderar toda la práctica docente y someterse a duros programas de formación.

D Finalidades y grandes objetivos que perfilan el carácter propio de la institución educativa concreta o del programa en cuestión.

D La disponibilidad de los recursos tecnológicos apropiados.

D Los elementos o características de la institución educativa o programa sobre los que se desea operar la innovación (toda la institución o programa o parte, con apoyos presenciales o sin ellos, con una u otra tecnología...) 
Resistencias, cambio y buenas prácticas en la nueva educación a distancia

Da dirección, grado o intensidad de la innovación. No es lo mismo presentar una innovación como suma o complemento a lo ya existente, que otra que pretenda sustituir o eliminar.

\subsection{LAS FUENTES DE RESISTENCIA AL CAMBIO}

En realidad son las personas quienes han de llevar a cabo las innovaciones. En nuestro caso es el docente concreto, con su determinada y única personalidad y los rasgos que lo caracterizan el que ha de acometer junto a otros el cambio. Por esto son importantes los factores de carácter personal que inciden en los mayores o menores deseos de innovar. Si esos factores están asumidos, de forma positiva o negativa, por grupos de docentes, su incidencia en la institución o programa, está garantizada, para bien, o para mal. Seleccionamos éstos (Marín y Rivas, 1984; García Aretio y Ruiz Corbella, 2001)

Due la innovación que pretende introducirse sea compatible, cercana o distante de los principios y valores de quienes han de ejecutarla.

Due se perciba o no con claridad una mejora futura,

D Que quienes han de innovar posean o no suficiente dominio sobre aquello que pretenden cambiar y sobre los pertinentes procesos.

Due los beneficios que se perciban superen o no a los costes.

- Que las cotas de incertidumbre que todo proceso innovador supone sean altas o bajas.

Partimos del supuesto de que intrínsecamente la innovación es buena, al menos en su pretensión, porque siempre trata de mejorar el sistema o institución. Vencer las resistencias al cambio debe ser un objetivo de los individuos y grupos innovadores Pero no por esto despreciamos los movimientos de resistencia y los obstáculos que existen para hacer efectiva una innovación, más al contrario, los valoramos porque de esta manera, si se vencen todos los obstáculos y se llega a implantar, siempre habrá más garantías de que la innovación desarrollada era la mejor.

Los individuos, grupos y elementos de resistencia ejercen realmente una importante labor de selección y filtro de deseos improvisados de cambiar porque sí, de freno a las ansias desmedidas de cambiar todo y deprisa. Esta función de filtro y regulación, garantiza la estabilidad e integridad de la institución o programa.

\subsection{AHORA EN EDUCACIÓN A DISTANCIA, ¿CAMBIAMOS POROUE «TOCA»?}

Estos que pretenden cambiar todo y deprisa, en muchos casos son individuos que han quedado prendados de las delicias de las tecnologías y suponen que 
las maravillas que se cantan de ellas diariamente a través de los medios de comunicación y de la calle, al aplicarlas, sin mayores análisis y estudios a la educación, supondrán el inmediato éxito en los logros de aprendizaje por parte de los estudiantes.

En fin, no sacralicemos las tecnologías porque en ningún ámbito social van a hacer milagros y, mucho menos, en el referido a la educación. Muchas profecías han caído con respecto a las implicaciones de las tecnologías en la educación. Recordemos que allá por los años 30 y 40, algunos programas radiofónicos se postulaban como sustitutos de algunas clases. ¿Qué decir de la televisión educativa que en los años 60 parecía que anunciaba la muerte de la escuela de aquella época? Las máquinas de enseñar (basadas en el conductismo-conexionismo) y aquellos rudimentarios ordenadores y sus posibilidades didácticas, también anunciaron la desaparición de los docentes.

El uso indiscriminado de tecnologías aplicadas a la educación, porque «toca» o están de moda, nunca es garantía de éxito, aunque sí un poderoso instrumento que bien utilizado por expertos tecnólogos de la educación puede producir excelentes resultados sobre la base de un determinado modelo pedagógico. Es decir, igual que se puede aplicar una determinada pedagogía autoritaria, reproductora, que fomente el pensamiento convergente, anule la capacidad crítica, etc., por procedimientos convencionales, igual de mal puede hacerse utilizando, por ejemplo, Internet. Y al revés, existen modelos de docencia presencial que fomentan una pedagogía liberadora, innovadora, imaginativa, al igual que los hay de este corte basados en Internet.

\subsection{RESISTENCIAS A LA INCORPORACIÓN DE LAS TECNOLOGÍAS}

Ciertamente, no todo debe ser aplausos para las tecnologías que se incorporan a la educación. Existen dificultades, problemas, inconvenientes, etc., de los que entresacar algunos de los que venimos detectando en la fecunda, exagerada, diversa y animosa floración de propuestas de instituciones, programas y cursos de educación a distancia, más o menos denominados como virtuales, sin aulas, online, de teleformación, e-learning, teleaprendizajelteleenseñanza, basados en la web/Internet, de enseñanza distribuida, etc., etc. Pretendemos en estudios posteriores (algún nuevo libro de futura aparición) penetrar en esta problemática, con el fin de sugerir posibles remedios a tantos males. Algunos defensores a ultranza de lo que denominan "nueva educación», cifran ésta en el uso indiscriminado de tecnología, deduciendo que ese cambio lo es también de las mismas bases o principios pedagógicos.

La realidad es que cada vez son más numerosas las instituciones (universitarias o no) que están introduciéndose en la aplicación de tecnologías para impartir una enseñanza no presencial. Y lo malo es que buena parte de estas insti- 
Resistencias, cambio y buenas prácticas en la nueva educación a distancia

tuciones presentan como indicador de calidad el uso de estas tecnologías, sin detenerse en verdaderos análisis de la calidad de sus aplicaciones, pertinencia y coherencia. En fin, enumeremos algunos de los peligros que venimos detectando e algunas aplicaciones de estas tecnologías al mundo de la educación que pueden suponer serios argumentos para la resistencia de determinados grupos:

D Es cierto, como ya afirmamos, que las tecnologías, Internet y la sociedad de la información, ofrecen inmensas posibilidades a la educación, pero la educación supone algo más que presentar un escaparate de informaciones. La educación, culmina, o debe pretender, la adquisición de conocimientos, sabiduría, habilidades, valores, hábitos, autonomía, libertad, sociabilidad..., y las tecnologías, Internet, la información, en sí mismos, no son educación.

D Los sistemas de información, por muy sofisticados que sean, no son per se sistemas de aprendizaje. Y existe la idea generalizada en muchos ámbitos empresariales e incluso docentes, de que difundir información y utilizar modernos sistemas de comunicación supone disponer de un sistema de aprendizaje, incluso para muchos, modélico y sobre todo actual o moderno.

D La pedagogía tradicional está muy arraigada en los cuadros de docentes y formadores como para propiciar cambios verdaderos, innovadores, basados en las tecnologías. El peligro será plasmar en la red un modelo de pedagogía reproductora y similar a la que ya se venía impartiendo en forma presencial.

D Son muchos los que piensan que por el hecho de utilizar tecnologías están renovando propuestas pedagógicas. En muchos casos lo que se hace es «colgar» en la red, sin la más mínima modificación y adaptación, las guías y textos impresos que ya se venían utilizando. La renovación siempre va a sustentarse en el saber, saber hacer pedagógico y actuar de los propios docentes. Los saberes, intenciones y prácticas del docente serán antes y más importante que el soporte tecnológico.

D Los docentes no están preparados, en muchos casos, para utilizar las tecnologías, ni siquiera a nivel de usuarios avanzados. Los estudiantes, generalmente van por delante con lo de situaciones ridículas que esta circunstancia puede generar.

D Se están llegando a soportar gran cantidad de acciones formativas en tecnologías, o en opiniones de los informáticos o expertos en red, en lugar de hacerlo en sólidos diseños proporcionados por la ciencia pedagógica o la Psicología.

- Con el objeto de mostrar las grandes posibilidades de la red, se llega a ofrecer a los estudiantes tal cantidad de información no debidamente seleccionada ni sistematizada que puede saturar, confundir y desorientar. Ni la escasez de información es buena, ni tampoco la sobreabundancia desordenada; como no lo es la pasividad total ni la atropellada hiperactividad; ni la sequía, ni tampoco la tempestad. 
D Se llega a confiar en exceso en el efecto motivador de las tecnologías. Ello puede valer para una motivación inicial, pero una sostenida motivación de mantenimiento requiere de algo más que navegar sin sentido por el maremagno de la red, visitando sitios más o menos estimulantes.

D Muchas propuestas asentadas en tecnologías avanzadas, no han previsto cuáles son las disponibilidades tecnológicas reales de los cuadros docentes y, sobre todo, de los destinatarios del programa.

D En numerosas instituciones se parte del absurdo presupuesto de suponer que los formadores están preparados, no sólo para usar la herramienta, sino para darle una aplicación didáctica a las mismas.

D La mayoría de las propuestas formativas apoyadas en tecnologías no parten de análisis rigurosos fundamentados en necesidades, disponibilidades, procesos, costes, previsión de resultados, etc.

D Se llegan a despreciar las tecnologías analógicas más convencionales, perdiéndose así posibilidades, si no más económicas, sí de mayor disponibilidad por parte de los destinatarios de la formación. Y, en todo caso, las tecnologías digitales incorporan e integran estas otras tecnologías que venía utilizando y sigue haciéndolo a veces con gran acierto, la educación a distancia de generaciones anteriores (García Aretio, 2001).

En fin, nuestro postulado, al pensar que la tecnología lo es todo, se ignoran los verdaderos principios pedagógicos que avalan las buenas prácticas educativas, sean éstas presenciales, a distancia (convencional) o basadas en Internet. Esos principios, como veremos después, poco cambiaron, aunque precisan de adaptaciones a las que obligan los ingentes recursos de que ahora disponemos.

No olvidemos que los medios, e Internet lo es, son sólo un elemento, instrumento o variable curricular más, muy significativos eso sí, pero una variable más al fin y al cabo, que deberemos movilizar cuando los objetivos, contenidos o problemas comunicativos a resolver así lo justifiquen (Cabero, 2001), y no por moda.

Además de los problemas enumerados, de componente más pedagógico, también existen otros a los que habrá de hacerse frente, referidos a la integración de las tecnologías en los procesos de educación a distancia, algunos de ellos, como podrá apreciarse, problemas que ya existían con la educación a distancia de corte más convencional:

D La falta de normatividad que regule las ofertas, fomente buenas prácticas y persiga el fraude.

D La resistencia a las prácticas de acreditación y evaluación como instrumentos esenciales para asegurar permanentemente la calidad y los procesos de reorganización de las instituciones a distancia. 
Resistencias, cambio y buenas prácticas en la nueva educación a distancia

D La falta de capacidad de las instituciones para transformar y actualizar su estructura organizativa, sus procesos de aprendizaje y sus programas de reclutamiento, formación y actualización de profesores, investigadores, directivos y administrativos.

Da desconfianza que aún muestran las autoridades de bastantes países ante esta modalidad educativa.

D La problemática que genera en autores y docentes todo lo relativo a la propiedad intelectual, derechos editoriales y de autor.

D El bajo nivel de cooperación interinstitucional.

D La escasez de acuerdos internacionales que permitan la homologación de títulos obtenidos en instituciones de otros países,

En fin, éstos y otros problemas y dificultades se presentan en ocasiones por falta de previsión, análisis de sus posibilidades, escasa preparación de los responsables de integrar estas tecnologías a los procesos de enseñanza y aprendizaje, etc. Aunque no olvidemos que a estas debilidades se le suman con demasiada frecuencia, como ya indicamos, las faltas de apoyos institucionales, gubernamentales y sociales.

\section{3. ¿QUÉ CAMBIA REALMENTE EN LA NUEVA EDUCACIÓN A DISTANCIA?}

Parece que Internet ha venido a confirmar y a reforzar todas las ventajas que los estudiosos del ODL (open and distance learning) asignábamos desde hace mucho tiempo a esa manera diferente de aprender (García Aretio, 1986, 1987a, 1994, 2001): apertura, flexibilidad, eficacia, privacidad y, sobre todo, interactividad. La economía de la educación a distancia con respecto a la de la educación cara a cara, que la venimos sustentando desde hace tiempo (García Aretio, 1985, 1987b), ha de matizarse. Si la queremos comparar con la impartida por instituciones que enseñan a distancia manteniendo sistemas organizados de tutoría presencial, la enseñanza a través de Internet, es más económica siempre que sustituya los sistemas tutoriales. Para instituciones de educación a distancia que venían ofertando cursos sin tutorías presenciales, la interactividad exigida por Internet, puede generar encarecimiento de costes.

Se llega a decir por personas ligadas a las nuevas instituciones o programas surgidos con el advenimiento de Internet, que ahora sí que puede ofrecerse calidad en estos procesos y que será difícil considerarlos, como se hacía antes, de "segunda fila». Es decir, parece que se piensa que los licenciados o egresados de universidades como la Open University británica, la UNED española, la FernUniversitaet alemana, el Centre Nacional d'Enseignement à Distance (CNED) 
de Francia, la Indira Ghandi National Open University (IGNOU) de la India, la University of South Africa (UNISA) de Sudáfrica, la Open Universiteit Nederland de Holanda, por nombrar sólo a unas cuantas, no estaban prestigiados antes de introducir en su metodología los aportes de Internet. Un licenciado de la Open University o de la UNED española, ambas con tres décadas de historia a sus espaldas, está más que probado que muestra índices de calidad en su formación, al menos similares a los manifestados por universitarios del sistema convencional. Parece que con aquellas afirmaciones se desea concluir que han cambiado, no sólo la distribución interna del edificio de la educación a distancia, sino sus propios cimientos.

Nosotros pensamos que de la clásica enseñanza por correspondencia al aprendizaje basado en la Web, ha cambiado sólo, y nada menos que, el soporte en el que se almacenan los contenidos y las vías de comunicación entre profesores y estudiantes y de estos entre sí, pero entendemos que buena parte de las bases teóricas de la educación a distancia, continúan siendo válidas, igualmente, para fundamentar procesos de enseñanza/aprendizaje soportados en Internet. ¿O es que aquello que define genéricamente a este tipo de educación ha cambiado? Nos estamos refiriendo a la «distancia»o separación sea ésta espacial y/o temporal.

Si venimos considerando que la educación a distancia se basa en un diálogo didáctico mediado entre el profesor (institución) y el estudiante que, ubicado en espacio diferente al de aquél, aprende de forma independiente (colaborativa) (García Aretio, 2001), tanto vale esa definición si el diálogo es real (producido a través del correo postal o electrónico, del teléfono o del chat) o simulado (sea a través del diálogo sugerido -conversación didáctica de Holmberg- en los materiales impresos o hipertextuales), síncrono (sea a través del teléfono o de la videoconferencia interactiva) o asíncrono (sea a través del correo postal o electrónico), soportado en papel, en formato electrónico o en la red. Queremos señalar con ello que pensamos que aunque las formas de soporte o almacenamiento de los contenidos y las vías o canales para la interacción, sean diferentes, las bases pedagógicas continúan vigentes, aunque algunas de ellas significativamente reforzadas. Es el caso del principio de interactividad, socialización o relación. Las tecnologías avanzadas no cambian ni entendemos que introduzcan nuevos principios pedagógicos en el ámbito del aprendizaje, otra cuestión será la referencia a la enseñanza.

Bien es cierto que si se hacen lecturas interesadas relativas a los más destacados y clásicos teóricos de la educación a distancia, tales como Otto Peters (1971 y 1993) que centró su aporte en el carácter de la forma industrial de enseñar y aprender que suponen estas propuestas; Wedemeyer (1971 y 1981) que centró su planteamiento en la independencia del estudio por correspondencia no sólo con respecto al espacio y tiempo sino también en su potencialidad de independencia en el control y dirección del aprendizaje; Moore $(1977,1990)$ que profundiza aspectos como los del diálogo y la estructura del diseño; Holmberg (1985) y su con- 
Resistencias, cambio y buenas prácticas en la nueva educación a distancia

versación didáctica guiada, podría concluirse, en algunos casos, que ahora podríamos hablar de un paradigma diferente, con bases diferentes y, naturalmente, con desarrollos también distintos. Si la lectura de estos teóricos es más reposada, podría pensarse, si no en todos, sí en buena parte de ellos, que sus deducciones son igualmente válidas para las realizaciones formativas soportadas en Internet.

Nosotros hemos realizado un serio intento por integrar todas estas teorías en la que venimos denominando como teoría del diálogo didáctico mediado (García Aretio, 2001). Propuesta que entendemos igualmente válida para procesos de enseñanza/aprendizaje a distancia de corte más convencional, como para aquellos que tienen como herramienta de almacenaje de contenidos y comunicación, a la red de redes.

Es importante resaltar que, aunque para muchos, parece que la educación a distancia acaba de nacer, esta modalidad educativa ha cumplido ya su siglo y medio de vida (García Aretio, 1999). Es decir, que no es nueva, que no es de hoy, aunque se la bautice con nuevas denominaciones, tales como: prefijo tele para referirse a la educación, la enseñanza o el aprendizaje, enseñanza y aprendizaje abiertos, enseñanza y aprendizaje virtuales, enseñanza y aprendizaje en red, aprendizaje electrónico, enseñanza mediada por ordenador, entorno virtual (de aprendizaje o colaborativo), etc. ¿Es el hecho de incorporar determinadas tecnologías lo que nos faculta para denominar su práctica incluso como nuevo paradigma educativo? Y como a la educación a distancia, desde su nacimiento, se le han ido incorporando tecnologías diferentes, ¿ es ahora, con Internet, cuando hemos de quitarnos el sombrero ante tan magno descubrimiento que cambia, según algunos, las propias bases de la educación?, ¿no será más cierto que estamos hablando de una evolución natural de la educación -siempre tan conservadora- como el que se produce en otros ámbitos de la vida, achacable en gran medida a la irrupción de Internet? (Holmber, 1998).

\section{1. ¿LOS MISMOS PRINCIPIOS O BASES?}

Tratemos de concretar nuestra idea relativa a algunos de los principios educativos básicos. Así, entendemos que principios como los del aprendizaje activo, colaborativo y las posibilidades de individualización o autonomía que ahora tanto se defienden como características propias del aprendizaje a través de Internet, figuran en las bases de la pedagogía contemporánea. Bien es verdad que ahora podemos afirmar que con estas tecnologías interactivas, estos principios son más alcanzables, pero en la enseñanza a distancia clásica, también podían y debían perseguirse. Y los teóricos de la educación a distancia los venimos defendiendo desde siempre.

Resulta aleccionador observar que desde instituciones que venían atacando (a través de sus docentes y directivos), o al menos ignorando, a la educación a dis- 
tancia que desarrollaban otras instituciones legalmente con la misma categoría y reconocimiento que aquellas, se muestran ahora las ventajas, posibilidades virtualidades, principios pedagógicos maravillosos de una nueva forma de enseñar $y$ aprender. Ignoran, más o menos intencionadamente, que esos principios también los mostraba la tan denostada enseñanza a distancia más convencional y menos virtual. Enumeremos algunos de éstos.

Da apertura se viene sosteniendo desde siempre entre los estudiosos y defensores de la educación a distancia. Ya decíamos que la educación a distancia diversificaba y ampliaba las ofertas de cursos para atender a la mayoría de necesidades actuales de formación, y que era posible impartir a la vez numerosos cursos desde la misma institución, sin problemas serios respecto al número de alumnos. También empleábamos esta característica para referirla a la atención de poblaciones dispersas. No es la apertura de esta forma de enseñar y aprender ago nuevo que hayamos de considerar con el advenimiento de Internet.

D La flexibilidad parece como si también se hubiese descubierto con los sistemas de eneñanza virtual. Eso de poder estudiar o acceder a la información dónde y cuándo se desee lo venimos afirmando desde nuestros primeros escritos sobre este tema. ¿ $\mathrm{O}$ es que no existía flexibilidad de espacio, tiempo, ritmo de aprender etc., cuando el estudiante podía consultar sus buenos (y a veces malos) textos impresos elaborados con una determinada tecnología, allá donde se encontrase y cuando le apeteciese, y sin necesidad de conexión alámbrica o inalámbrica ni de corriente eléctrica ni baterías?, y dígase algo similar para el estudio a través de audio o vídeo.

Da democratización del acceso a la educación ha venido siendo desde siempre uno de los objetivos básicos para la creación de instituciones y programas de educación a distancia. Reconocemos que con Internet, se ha democratizado el acceso a la información y, a través de la educación, al conocimiento, pero no puede defenderse que la democratización del acceso a la educación sea una característica distintiva de la educación a través de Internet, dado que ya lo era de la educación a distancia de corte más convencional. Y, además, no olvidemos que para esa democratización real, ahora se profundiza la denominada brecha digital, entre los ciudadanos, sectores, regiones o países con más y con menos posibilidades de acceder a estas tecnologías.

D La socialización y la interactividad. El aprendizaje colaborativo, el cooperativo, el tutelado entre los propios alumnos, denominados todos ellos por Perkins (1997) como educación entre pares, se ha venido fomentando, por ejemplo, en instituciones a distancia de gran prestigio nacidas en la década de los años 70 del pasado siglo, a través de las tecnologías más convencionales tales como el teléfono, el correo postal y las audioconferencias y, sobre todo, a través de las sesiones de tutoría presencial y de la conforma- 
Resistencias, cambio y buenas prácticas en la nueva educación a distancia

ción de grupos de trabajo compuestos por estudiantes residentes en zonas geográficas cercanas. ¿Es que el estudiante no podía conectar telefónicamente con el docente a las horas especificadas por éste, al igual que esas horas especificadas se establece ahora una sesión de chat?, ¿o es que el estudiante a distancia de antes no podía enviar recibir una carta en cualquier momento y desde cualquier lugar a su docente o compañeros de estudio?, ¿o eso mismo, dónde y cuándo quisiese, no lo podía hacer mediante el fax? Ciertamente los entornos virtuales (que podríamos entender como la interacción a través de medios telemáticos) pueden hacer más inmediata, fácil y frecuente esta interacción. Pero ya había, también antes, interacción a través de otros medios y modelos.

Dl Erincipio de actividad, debe ser consustancial a cualquier propuesta educativa realizada mediante cualquier modalidad. En la educación a distancia convencional se trataba de un principio de gran relieve que ahora puede verse también potenciado por el uso de las herramientas tecnológicas más avanzadas. El estudiante, como sujeto activo de su propio proceso de construcción del aprendizaje, es más protagonista en la enseñanza a distancia que en la enseñanza presencial. Y ese protagonismo se mantiene y probablemente se aumenta en la formación en espacios virtuales.

- El principio pedagógico de la individualización ya defendido, al igual que los dos anteriores, por la Escuela Nueva de finales del siglo XIX, ya era una clara apuesta de la educación a distancia más convencional. La educación en espacios virtuales puede potenciarla pero no la ha descubierto. La libertad e independencia en el estudio o, si mejor queremos hablar, de las posibilidades y ventajas del autoaprendizaje, ya se daban y trataban de destacarse en la enseñanza a distancia «no virtual». La lectura negativa de este principio nos llevaría al individualismo, tan deplorable en cualquier tipo o modalidad educativa. ¿O es que no se están generando también grandes problemas de soledad y escasa socialización cuando tanto se depende de Internet? De la socialización virtual a la soledad presencial.

- La motivación o fascinación ante el ordenador, como argumento de la facilidad de aprender a través de un medio que divierte, atrae y estimula, tampoco es nuevo. Ya en la enseñanza presencial existían determinados recursos: mapas, dibujos, instrumental de laboratorio, diapositivas, filminas que fascinaban a nuestros alumnos. La radio, la televisión, el audio, el vídeo, los atractivos textos impresos de numerosos programas a distancia, ya suponían un acicate para el estudio.

Pero, si damos un paso más y nos adentramos en teorías o principios más actuales, observamos que las bases ya eran sólidas antes de la irrupción de Internet. Para muchos parece como si el constructivismo hubiese nacido para Internet y viceversa. ¿Es que no se producía aprendizaje de corte conductista antes de los estudios de Pavlov y, de Skinner? Lo mismo podríamos señalar de las restantes teorías 
del aprendizaje. Son todos éstos, enfoques diferentes relativos a la visión que pueden tener los docentes sobre cómo pueden aprender mejor sus alumnos. No cabe duda de que las tecnologías pueden aupar los beneficios de unos enfoques sobre otros, pero nadie negará que con las más avanzadas tecnologías se pueden seguir aplicando teorías del aprendizaje de la más rancia escuela. Queremos destacar que principios constructivistas pueden (y deben) ponerse en práctica en entornos presenciales, en entornos de educación a distancia sin Internet y, naturalmente, en entornos virtuales ubicados en la red que muestran innumerables ventajas.

Si quisiéramos resumir los rasgos de estas formas de enseñar y aprender y destacar cuáles de ellos son más propios o han sido potenciados con la integración de las tecnologías en este tipo de educación, nos encontraríamos con la siguiente tabla ${ }^{1}$ :

\begin{tabular}{|c|c|c|c|}
\hline Rasgo & Características & $\begin{array}{l}\text { Ead } \\
\text { Conv.* }\end{array}$ & $\begin{array}{l}\text { Ead } \\
\text { TIC* }\end{array}$ \\
\hline Apertura & $\begin{array}{l}\text { A diversa y amplia oferta de cursos. } \\
\text { A destinatarios dispersos. } \\
\text { A diferentes entornos, niveles y estilos de aprendizaje. } \\
\text { A las necesidades actuales. } \\
\text { A la segunda oportunidad. }\end{array}$ & $\mathrm{x}$ & + \\
\hline Flexibilidad & $\begin{array}{l}\text { De espacios ¿dónde estudiar? } \\
\text { De tiempo ¿cuándo estudiar? } \\
\text { De ritmos ¿a qué velocidad estudiar? } \\
\text { Para combinar familia, trabajo y estudio } \\
\text { Para permanecer en el entorno familiar y laboral } \\
\text { Para compaginar estudio con otras alternativas. }\end{array}$ & $\mathrm{X}$ & + \\
\hline \multirow[t]{4}{*}{ Eficacia } & Posibilidad de aplicar con inmediatez lo que se aprende & $\mathrm{x}$ & $=$ \\
\hline & Integración de medios para aprender. & & \\
\hline & Autoevaluación de los aprendizajes. & & \\
\hline & Posibilidad de que mejores especialistas elaboren materiales. & & \\
\hline Economía & $\begin{array}{l}\text { Al obviar pequeños grupos. } \\
\text { Al ahorrar gastos de desplazamiento. } \\
\text { Al evitar abandono de puesto de trabajo. } \\
\text { Al disminuir tiempo complementario de permanencia en trabajo } \\
\text { Al propiciar la economía de escala }\end{array}$ & $\mathrm{x}$ & - \\
\hline $\begin{array}{l}\text { Formación } \\
\text { Permanente }\end{array}$ & $\begin{array}{l}\text { Al dar respuesta a la gran demanda de formación. } \\
\text { Al mostrarse como ideal para la formación en servicio. } \\
\text { Al propiciar la adquisición de actitudes, intereses y valores. }\end{array}$ & $\mathrm{x}$ & $=$ \\
\hline
\end{tabular}

${ }^{1}$ Extraída de lo ya publicado en García Aretio, L. (2001). La educación a distancia. De la teoría a la
práctica. Barcelona: Ariel. 
Resistencias, cambio y buenas prácticas en la nueva educación a distancia

\begin{tabular}{|c|c|c|c|}
\hline Rasgo & Características & $\begin{array}{l}\text { Ead } \\
\text { Conv. }\end{array}$ & 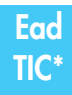 \\
\hline Privacidad & $\begin{array}{l}\text { Al propiciar la posibilidad de estudiar en la intimidad. } \\
\text { Al evitar lo que para muchos puede suponer la presión del grupo. } \\
\text { Al invitar a manifestar conocimientos o habilidades que ante } \\
\text { el grupo se evitarían. }\end{array}$ & $X$ & $=$ \\
\hline Interactividad & $\begin{array}{l}\text { Al hacer posible la comunicación total, bidireccional y } \\
\text { multidireccional. } \\
\text { Al hacerla más próxima e inmediata. } \\
\text { Al posibilitar la interactividad puede síncrona o asíncrona. }\end{array}$ & $x$ & ++ \\
\hline $\begin{array}{l}\text { Aprendizaje } \\
\text { activo }\end{array}$ & $\begin{array}{l}\text { Estudiante es sujeto activo de aprendizaje. } \\
\text { El autoaprendizaje exige en mayor medida la actividad }\end{array}$ & $x$ & $=$ \\
\hline $\begin{array}{l}\text { Apr. } \\
\text { Colaborativo }\end{array}$ & $\begin{array}{l}\text { El aprender con otros, de otros y para otros, sea este } \\
\text { aprendizaje de forma más o menos guiada (cooperativo). }\end{array}$ & $x$ & ++ \\
\hline $\begin{array}{l}\text { Macro- } \\
\text { información }\end{array}$ & $\begin{array}{l}\text { Al poner a disposición la mayor biblioteca jamás imaginada. } \\
\text { Ninguna biblioteca de aula, centro o universidad alberga tantos } \\
\text { saberes como los depositados en la red. }\end{array}$ & 0 & $x$ \\
\hline $\begin{array}{l}\text { Recuperación } \\
\text { inteligente }\end{array}$ & $\begin{array}{l}\text { Al propiciar que el estudiante pase de receptor de información } \\
\text { a poseer la capacidad de buscar, seleccionar y recuperar } \\
\text { inteligentemente la información. }\end{array}$ & 0 & $x$ \\
\hline $\begin{array}{l}\text { Democrati- } \\
\text { zación } \\
\text { educación }\end{array}$ & $\begin{array}{l}\text { Al superar el acceso limitado a la educación por razones } \\
\text { laborales, de residencia, familiares, etc. }\end{array}$ & $x$ & $=$ \\
\hline $\begin{array}{l}\text { Democrati- } \\
\text { zación de } \\
\text { información }\end{array}$ & $\begin{array}{l}\text { Al hacer realidad la universalidad de la información. } \\
\text { Todos pueden acceder a todo tipo de documentos textuales y } \\
\text { audiovisuales. }\end{array}$ & 0 & $x$ \\
\hline $\begin{array}{l}\text { Diversidad y } \\
\text { dinamismo }\end{array}$ & $\begin{array}{l}\text { La información es diversa, variada y complementaria. } \\
\text { La web ofrece múltiples maneras de acceder al conocimiento } \\
\text { de forma variada y dinámica. }\end{array}$ & 0 & $x$ \\
\hline Inmediatez & $\begin{array}{l}\text { La respuesta ante las más variadas cuestiones se ofrece a gran } \\
\text { velocidad, al margen de la hora y el lugar. }\end{array}$ & 0 & $x$ \\
\hline Permanencia & $\begin{array}{l}\text { La información no es fugaz como la de la clase presencial, } \\
\text { la emisión de radio o televisión. } \\
\text { El documento hipermedia está esperando siempre el momento } \\
\text { adecuado para el accedo de cada cual. }\end{array}$ & - & $x$ \\
\hline Multiformatos & $\begin{array}{l}\text { La diversidad de formatos en los que puede ofrecerse } \\
\text { la información estimula el interés por aprender y puede ofrecer } \\
\text { ángulos diferentes del concepto, idea o acontecimiento. }\end{array}$ & - & $x$ \\
\hline $\begin{array}{l}\text { Multidireccio- } \\
\text { nalidad }\end{array}$ & $\begin{array}{l}\text { Existe gran facilidad para que documentos, opiniones y } \\
\text { respuestas tengan simultáneamente diferentes y múltiples } \\
\text { destinatarios, seleccionados a golpe de «clic». }\end{array}$ & 0 & $x$ \\
\hline
\end{tabular}




\begin{tabular}{llcc}
\multicolumn{1}{c}{ Rasgo } & \multicolumn{1}{c}{ Corracterísticas } & $\begin{array}{c}\text { Ead } \\
\text { Conv** }\end{array}$ & $\begin{array}{c}\text { Ead } \\
\text { Teleubicuidad }\end{array}$ \\
& $\begin{array}{l}\text { Todos los participantes en el proceso de enseñanza y aprendizaje } \\
\text { pueden estar virtualmente presentes en muchos lugares a la vez. }\end{array}$ & 0 & $\mathrm{X}$ \\
\hline $\begin{array}{l}\text { Libertad } \\
\text { edición y } \\
\text { difusión }\end{array}$ & $\begin{array}{l}\text { Todos pueden editar sus trabajos y difundir sus ideas que pueden } \\
\text { ser conocidas por multitud de internautas. }\end{array}$ & 0 & $\mathrm{X}$ \\
\hline $\begin{array}{l}\text { Interdiscipli- } \\
\text { nariedad }\end{array}$ & $\begin{array}{l}\text { Todos los ángulos, dimensiones y perspectivas de cualquier } \\
\text { cuestión pueden ser contemplados desde diferentes áreas } \\
\text { disciplinares y presentados de manera inmediata. }\end{array}$ & 0 & $\mathrm{X}$ \\
\hline
\end{tabular}

(*) Leyenda:

Ead Conv. - Educación a distancia convencional

Ead TIC - Educación a distancia basada en la red.

$X$. Rasgo es propio de una u otra.

0 . Rasgo no poseído.

-. Rasgo manifestado en menor orden.

+. Rasgo manifestado en mayor proporción.

\section{2. ¿QUÉ CAMBIA ENTONCES?}

Como puede observarse, rasgos fundamentales y definitorios de la educación a distancia, permanecen en ambos modelos, en algunos casos potenciados. Otras características que entendemos no sustanciales desde una perspectiva conceptual, aunque sí muy relevantes y favorecedoras de esta forma de enseñar y aprender, son igualmente destacados en la tabla precedente.

No reside en el uso de las tecnologías, por tanto, el cambio de modelo pedagógico. Este cambio siempre dependerá de la formación, intención y decisión del educador, del pedagogo, y no del experto informático o especialista en redes. De estos últimos, sin duda va a depender buena parte del éxito de una propuesta soportada en la red pero ellos y sus tecnologías, por sí mismos, nunca garantizarán el éxito. Y en muchos casos, ellos nos van a ofrecer tecnologías que no deseamos o que no cubren nuestras expectativas y necesidades como pedagogos. ¿De qué nos sirven excelentes diseños de alto nivel y complejidad tecnológica y de estética incuestionable si los contenidos que en ellos se soportan son pobres, llenos de errores, con escasa estructura didáctica o poco pensados para la índole y nivel del curso o para las necesidades experiencias de los destinatarios?

Es decir, que más que considerar un cambio radical en las bases teóricas que conforman estas estrategias de enseñanza y aprendizaje activos y cooperativos, deberíamos poner el acento en la capacidad de Internet para permitir un elevado nivel en calidad, cantidad y rapidez de la interactividad, y para facilitar acceso a 
Resistencias, cambio y buenas prácticas en la nueva educación a distancia

un inmenso volumen de información, aunque parte considerable de ella, de contenido muy mediocre e, incluso, deplorable. En efecto, Internet, las tecnologías avanzadas, pueden favorecer aprendizajes de mayor calidad pero nunca garantizarlos. Al igual que utilizando otras tecnologías menos sofisticadas, más sencillas y económicas se pueden alcanzar metas de aprendizaje de calidad. Conviene no olvidar la búsqueda de eficiencia de los diferentes programas, es decir, la relación coste/eficacia.

En fin, ¿qué cambia entonces? Entendemos que cambian con los nuevos aportes tecnológicos, las estrategias de enseñanza-aprendizaje, la metodología, los recursos y su organización, los sistemas de comunicación, la distribución de materiales de estudio, posiblemente la eficiencia, que no siempre la eficacia, las posibilidades de acceso y la universalización o democratización del acceso, que ya supuso un gran avance con la educación a distancia de corte más convencional.

Pero al margen de que los cambios los consideremos más o menos sustanciales o más o menos radicales, la realidad es que esas tecnologías a las que aludíamos al principio de este trabajo, están ahí y desde la perspectiva educativa ofrecen grandes posibilidades para el logro de metas de aprendizaje de alta calidad. Y, sin duda, nos van a obligar a replantearnos o redefinir: la organización y la planificación de los procesos educativos, el diseño y desarrollo de los materiales de formación, los instrumentos y el proceso de evaluación, las relaciones horizontales y verticales, etc. (Gisbert, 2000).

\section{DE LAS RESISTENCIAS Y DIFICULTADES, A LA MEJORA}

Ya somos conscientes de la necesidad de soportes teóricos sólidos para las buenas prácticas, conocemos la posible procedencia, dirección e intensidad de las resistencias a la incorporación de las tecnologías avanzadas a la educación a distancia y, serenamente, hemos aterrizado en los límites de lo que realmente cambia o puede cambiar en la nueva educación a distancia.

Pues bien, bueno será seleccionar una serie de aciones prioritarias que se nos antojan como esenciales si, ciertamente, se pretende la mejor calidad en las prácticas de educación a distancia, ahora que Internet la afecta de forma determinante.

\subsection{LA INVESTIGACIÓN}

Necesitamos incrementar una investigación que ofrezca pistas y argumentos para la toma de decisiones que refuercen los procesos y buenas prácticas de la enseñanza y el aprendizaje a través de Internet. Aunque los fundamentos o marco teórico de la educación a distancia decíamos que han cambiado poco, no existe el adecuado soporte teórico que suponga una base sólida en la que apoyar pro- 
cedimientos, estrategias y buenas prácticas de enseñanza/aprendizaje a través de Internet.

Pero para ello, convencidos de que ha de darse racionalidad científica, base teórica, a la educación a distancia, precisaríamos conocimientos coherentes, sistemáticos, y ordenados de distinta índole. Y si entendemos cómo están organizados estos conocimientos, estamos construyendo una teoría, porque este sistematizado cuerpo de ideas, conceptos y modelos nos debería ayudar a construir significados, explicar, interpretar y comprender la acción. Elaborar ese necesario marco teórico en el ámbito de la educación a distancia, nos llevaría a observar los aspectos a los que se refiere esta realidad educativa y reflexionar sobre ellos. ¿Qué aspectos?:

D rasgos que definen a la educación a distancia,

Dus componentes,

D cómo son y se relacionan entre sí estos componentes,

Dué principios los sostienen,

Dué normas generan,

D cómo se aplican éstas y por qué,

Dué sucederá si se aplican de una u otra manera, etc.

No se trataría, por tanto, sólo de describir, especular y reflexionar sobre el fenómeno innovador, sino de observar y comprender los hechos empíricos que muestra esta práctica educativa, como fuente para sistematizar el cómo hacer tecnológico en enseñanza a distancia y el propio qué hacer, e incluso la predicción (qué será o qué podría ser) (Garrison, 2000), con el fin de reelaborar los principios, leyes y normas que posibilitan la buena práctica de una forma de enseñanza no presencial.

Dando un paso más, y asumidas esas bases teóricas, se haría preciso abordar vastos y urgentes desarrollos en investigación e innovación de programas con estructuras diferentes a los convencionales y que al relacionarlos con la educación a distancia basada en Internet, supondría:

Cómo mejor enseñar y aprender a través de estas tecnologías.

D Con qué objetivos, contenidos y a qué ritmos.

D Cómo establecer las relaciones virtuales ideales entre docentes y estudiantes.

D Cómo planificar las diferentes acciones formativas en entornos virtuales.

D Cómo organizar y gestionar las distintas unidades de un centro, institución o programa de estudios soportado en la red.

D Cómo organizar la participación de los diferentes sectores de la comunidad de estudio. 
Resistencias, cambio y buenas prácticas en la nueva educación a distancia

D Cómo evaluar los aprendizajes, cómo hacerlo a los docentes, programas, instituciones y a las propias tecnologías.

En suma, se precisa invertir en investigaciones y estudios de calidad que nos alumbren sobre la escuela, la universidad, la educación que viene. Han de elaborarse hipótesis futuristas sobre cómo vamos a educar en los próximos años que, evidentemente, no va a ser como lo hemos venido haciendo el siglo pasado, aunque seguiremos haciendo eso, educación.

Somos conscientes de que las administraciones públicas suelen despreciar o valorar escasamente estas necesarias investigaciones por lo que existen pocas previsiones de fondos económicos para desarrollarlas. Además, sabemos de la dificultad que comporta este tipo de investigaciones en las que se relacionan aspectos de carácter tecnológico con otros de componente pedagógico.

Por otra parte, los resultados de las investigaciones pocas veces son considerados, ni siquiera por aquellas instituciones u organismos que las encargaron y subvencionaron. Cuánto más decir de lo lejos que, habitualmente, quedan del docente común. Este docente no suele tener acceso a este tipo de trabajos que, además, son poco asequibles y comprensibles para ellos.

En fin, nos reafirmamos en que, no pueden existir avances serios en el campo de la educación y formación en espacios virtuales si no están sustentados en estudios e investigaciones que concluyan con recomendaciones para el desarrollo de mejores prácticas.

\subsection{LA FORMACION DE RECURSOS}

Una segunda necesidad hace referencia a la formación de los recursos humanos, directivos, profesores, técnicos y administrativos. Nos referiremos especialmente a la más importante para nosotros, la formación de docentes. Porque los docentes son el componente esencial que puede concretar la acción formativa según el contexto, los destinatarios, la índole de la materia o curso y la disponibilidad de determinados recursos. Son la piedra angular sobre la que pivota todo cambio.

Por eso, los gobiernos, las instituciones educativas y de formación deben priorizar una preocupación cada vez más aguda, conforme avanza el mundo de la tecnología, la capacitación del profesorado. Los profesores son la clave para un aprendizaje de calidad en general y soportado en la Web, en particular. Si los docentes no adquieren las estrategias básicas para el diseño de cursos, o, al menos, para la gestión de los mismos, por muy bueno que sea el entorno virtual de aprendizaje, su fracaso está anunciado. La voluntad de asumir una determinada propuesta innovadora y acometerla, por parte del profesorado es imprescindible si no desea convertir cualquier innovación o reforma en letra muerta. 
Y la realidad nos muestra que la mayoría de los docentes no están preparados para usar las tecnologías en la enseñanza. Fueron educados sin ordenador y sin Internet y piensan que no fueron mal formados. Vienen educando a sus alumnos a distancia de una determinada manera más o menos convencional y se preguntan, ¿por qué hacerlo de otra forma?

De manera que una labor inicial de los gobiernos e instituciones será la de convencer a sus docentes de las ventajas que pueden suponer las tecnologías avanzadas aplicadas a procesos de formación a distancia y qué se puede hacer con esa tecnología. Posteriormente, no antes, se deberá proceder a un plan de formación atractivo, bien diseñado y, naturalmente, realizado a través de estas herramientas electrónicas, con el fin de que estos docentes sean los artífices esenciales de esta transformación educativa, dado que si saben lo que se puede hacer con las TIC, pueden reflexionar sobre cómo pueden ser utilizadas para mejorar la educación.

No cabe duda que se ha hecho un esfuerzo en formar sobre las herramientas tecnológicas, sin embargo se ha hecho muy poco en formaciones significativas sobre prácticas pedagógicas innovadoras (COM, 2000a). El cambio pedagógico no vendrá por la mera aplicación de las tecnologías, éste vendrá cuando los docentes sean conscientes de todas sus posibilidades y vinculen adecuadamente las tecnologías a la práctica pedagógica de cada una de las disciplinas del currículo. El énfasis no ha de ponerse en la disponibilidad y potencialidades de las tecnologías sino en los cambios de estrategias didácticas de los docentes en cuanto a el diseño y estructura de los propios contenidos y a los sistemas de comunicación tanto verticales como horizontales (Salinas, 2000).

Ciertamente aparecen nuevas competencias docentes con la integración de las tecnologías en los procesos educativos y también se modifican otras que ya venían desarrollando los profesores de la educación a distancia convencional. Si hacemos un intento por resumir dónde deberían concentrarse algunos esfuerzos de formación de docentes, podríamos apuntar hacia el desempeño de calidad de una serie de tareas que se nos antojan como básicas:

Diseñador, o al menos gestor, del curso. Planificador y organizador de recursos (Inglis, 1999).

D Proveedor de fuentes de información. Que supone una cualificación para buscar, seleccionar, procesar, valorar, estructurar informaciones y conocimientos y formar a los alumnos en estas técnicas.

D Facilitador que descubre caminos, apunta estrategias de aprendizaje y soluciona problemas y dudas.

D Promotor de temas de debate, trabajos colaborativos, estudio de casos, guía y moderador de los debates escritos o verbales (chat o videoconferencia).

D Evaluadores, con incidencia en la modalidad formativa de la evaluación. 
Resistencias, cambio y buenas prácticas en la nueva educación a distancia

D Orientador, tutor y creador de un ambiente agradable para el aprendizaje. Ello supone el seguimiento del progreso del estudiante, la permanente disponibilidad y motivación, la atención a la configuración de los diferentes grupos de trabajo, etc.

Las tareas o funciones señaladas, dependerá del modelo de educación a distancia que se siga, se concentrará en una o más personas componentes del equipo docente. En determinados modelos la última de las funciones señaladas las desempeña una persona diferente a la(s) que desarrolla(n) las restantes tareas (García Aretio, 2001).

En fin, no olvidemos la importancia que también tiene la formación de los otros recursos que completan los equipos de personas responsables de las institucones, programas y cursos, los directivos, gestores, técnicos, administrativos, etc.

\subsection{LA PLANIFICACIÓN}

La complejidad del proceso educativo requiere de una racionalización del mismo. El enlace entre la teoría educativa y la práctica exige la articulación de todos los elementos, estructuras y fases propios de una planificación o matriz para la intervención.

Nosotros entendemos la planificación como el diseño de un plan ordenado, coherente, sistemático y secuencial de todos los elementos o factores que intervienen o pueden intervenir en una acción formativa que pretende unos objetivos o propósitos con el fin de resolver un problema o atender a una determinada necesidad de formación en una realidad concreta (García Aretio, 1994).

Y esta formulación nunca habrá de ser cerrada, sino abierta y provisional, y ello porque:

D los propios fines pueden cambiar durante el proceso, o

D puede existir inconsistencia o discrepancia entre algunos elementos, o

D la información que poseemos, al irse ampliando, ofrece nuevas pistas para reconducir el programa. tados:

Optamos por un modelo que diversifica la planificación en tres grandes apar-

D diseño en el que incluimos la fundamentación o condicionamientos de la planificación y la especificación o concreción de ese diseño;

D desarrollo que comprende la producción de los materiales y la aplicación del proceso propiamente dicho y, por fin,

D evaluación de todas y cada una de las fases de la planificación. 


\subsection{LA EVALUACIÓN PERMANENTE}

Si de evaluar la calidad de una institución o programa educativo o de formación se trata, estaremos hablando de evaluación institucional o de intento de valorar el grado de consecución de las metas y objetivos propuestos, así como las condiciones de partida y los procesos (Weert, 1990). Esta evaluación, por una parte puede ser llevada a cabo por la propia institución (evaluación interna) a través de alguna comisión, departamento, o instituto de la propia entidad con objeto de mejorar el producto, o mediante alguna comisión exterior u organismo especializado imparcial. Igualmente unas evaluaciones se centran en la valoración del grado de consecución de los objetivos (eficacia) o del grado de aprovechamiento de los recursos en función de los objetivos (eficiencia). Ciertas evaluaciones se ocupan de los procesos, otras de los resultados y otras de la relación existente entre las metas propuestas y la disponibilidad de recursos para su logro. Por fin, todas las evaluaciones, si pretenden mejorar la calidad del producto deberían culminarse con la innovación necesaria para el logro de ese producto de mejor calidad.

Basados en estas reflexiones vamos a esbozar un modelo que entendemos integrador, basándonos en las características que refería la National Commission on Excelence in Education en 1983 (García Aretio, Medina Rubio, López-Barajas y Marín Ibáñez, 1991) como propias delimitadoras del principio de calidad, a las que nosotros añadimos otras tres que perfilarán el modelo (García Aretio, 1994). Por tanto, en este catálogo de ajustes o de relaciones de coherencia, concordancia, congruencia o armonía basamos nuestro concepto de calidad de la docencia.

Funcionalidad: Coherencia entre objetivos, metas y resultados educativos y el sistema de valores, expectativas y necesidades culturales y socio-económicas de una Comunidad. Se trataría de la funcionalidad cultural y socio-económica de la institución. Desde esta perspectiva la disfuncionalidad, inadaptación o poca calidad de una institución o de un programa, haría referencia a que sus objetivos, componentes internos y resultados pueden ser teóricamente buenos y coherentes entre sí, pero no sirven para dar solución al problema o necesidades sociales en función de los cuales se establecen.

Eficacia o efectividad: Coherencia entre metas y objetivos educacionales considerados como valiosos y deseables en la institución o programa, y los resultados alcanzados. Se pueden haber obtenido unos resultados no necesariamente malos y, sin embargo, al no corresponderse con los objetivos y metas deseados y esperados, fallar la eficacia de la institución.

Eficiencia: Coherencia entre entradas, procesos y medios y los resultados educativos (relación entradas/medios-producto). Eficiencia entendida no solo en una acepción económico-administrativa (máximos resultados con mínimos costos, o índice de productividad), sino también pedagógica, como adecuación y va- 
Resistencias, cambio y buenas prácticas en la nueva educación a distancia

lidez de las estrategias de intervención, tiempos, recursos instructivo y curriculares... a unas metas de educación. Es decir, pueden lograrse las metas y objetivos propuestos pero a un alto coste humano, material o económico que rebajaría el grado de eficiencia.

Como antes indicábamos, a estas tres características, nosotros agregaríamos las siguientes:

Disponibilidad: Coherencia entre las metas y objetivos propuestos y los recursos humanos, materiales y económicos de que puede disponerse para iniciar el proceso. Sería la relación entre las metas y las entradas. De poco serviría formular, incluso de forma consensuada, unas metas y objetivos valiosos e, incluso, unos procedimientos o procesos acertados si no se cuenta con infraestructura, personal y presupuesto adecuados.

Información: Coherencia entre los resultados obtenidos y las propuestas de mejora que se ofrecen en el pertinente informe. Las conclusiones del informe deben ser coherentes con las sugerencias de mejora consistentes en reforzar los puntos fuertes y combatir los débiles. Este informe, según los términos que se acuerden, debe difundirse adecuadamente. De nada serviría haber obtenido determinados resultados si de ellos no se extrae el correspondiente informe. En él se rinden cuentas y se ofrecen las bases para la mejora de la institución.

Innovación: Coherencia entre el catálogo de mejoras precisas (reforzamiento de puntos fuertes y corrección de los débiles) para el logro de las metas, y la decisión de innovar y revisar, bien esas metas -podrían ser poco realistas- o con más seguridad, las entradas y los procesos. Poco avanzaríamos en el modelo si, contando con todos los ajustes antes señalados, la evaluación no tuviese reales consecuencias para la mejora del sistema, innovando metas, entradas o procesos en los que se hubieran detectado puntos débiles.

En fin, entendemos que las redes, organismos o subsistemas, deberán contener los mecanismos necesarios para definir y aplicar procesos continuos y modernos de evaluación y acreditación para cursos, programas e instituciones. La acreditación y evaluación son dos instrumentos esenciales para asegurar permanentemente la calidad y los procesos de reorganización de las instituciones a distancia. Por ello apostamos porque se lleven a cabo en todas las instituciones procesos de evaluación institucional de carácter interno que sean complementados con la pertinente evaluación de organismos externos a cada institución.

\subsection{REDES INSTITUCIONALES Y PERSONALES}

Para sustituir las acciones aisladas, dispersas e ineficaces de instituciones y programas a distancia, entendemos que existen en la actualidad posibilidades pa- 
ra crear o consolidar, las ya existentes, redes o subsistemas de educación a distancia, conformados por instituciones públicas y privadas, aprovechando el potencial de las nuevas tecnologías. La voluntaria inserción en cada red debería suponer el compromiso de cumplir con los procesos de evaluación y acreditación que se propongan.

Además de las redes de carácter institucional, deseamos destacar la conveniencia de que las personas más implicadas en los procesos de educación a distancia se nutran de la información y documentación que hoy pone a disposición de todos Internet y participen en los foros de intercambio, de trabajo y aprendizaje colaborativo existentes. Un ejemplo para atender esta recomendación, puede ser el Centro Iberoamericano de Recursos para la Educación a Distancia de la Web de la Cátedra UNESCO de Educación a Distancia de la UNED: www.uned.es/cued.

\subsection{LOS APOYOS POLÍTICOS}

De poco serviría todo lo anteriormente expuesto si encontramos oposición o indiferencia en ámbitos institucionales (ya hicimos referencia a las resistencias), sociales (percepción que tiene la sociedad sobre estas formas de enseñar y aprender) y políticos.

Referente a estos últimos iríamos más allá de los meros apoyos. Entendemos que los organismos internacionales y los gobiernos no pueden ignorar la incidencia que las tecnologías están teniendo en el mundo de la educación a distancia. La explosión de propuestas de educación a través de la red, la multiplicación de empresas e instituciones que ofrecen software, plataformas virtuales completas, cursos, etc, exigen a las administraciones públicas determinados compromisos, que amparen a los que más necesitan del bien de la educación; que cubran las lagunas normativas en este campo; que impulsen la homologación de títulos; que amparen la propiedad intelectual; que propicien la vigilancia crítica de los medios, y que favorezcan el establecimiento de consorcios y redes.

El esfuerzo de quienes trabajamos en esta área de estudio debe ser, en buena parte, el de mostrar a políticos y líderes sociales las posibilidades de esta forma de enseñar y aprender, exigiéndoles apoyos y compromisos que puedan redundar en las prácticas de más calidad que hemos tratado de defender en este trabajo.

\section{CONCLUSIONES}

Acabamos como empezamos este trabajo, en este caso concluyendo con los objetivos de acción que nos propndríamos para la mejora de las prácticas en educación a distancia: 
Resistencias, cambio y buenas prácticas en la nueva educación a distancia

1. Entender que las mejores prácticas siempre se apoyan en buenas propuestas teóricas y no en modas.

2. Aprender, para vencer las resistencias personales, endógenas y exógenas al cambio.

3. Asumir que los clásicos principios pedagógicos siguen vivos en las nuevas propuestas de enseñanza y aprendizaje virtuales.

4. Valorar los aportes de las tecnologías a las prácticas educativas de calidad, sin olvidar las dificultades y problemas que comporta su uso indiscriminado.

5. Acomenter rigurosos procesos de investigación para la mejora de la educación a distancia de hoy.

6. Impulsar permanentes procesos de formación de los equipos docentes y de todo el personal involucrado.

7. Plasmar cuidadosas planificaciones que supongan una guía para la reflexión y la acción.

8. Someter a las instituciones, programas y docentes a los correspondientes procesos de evaluación de la calidad.

9. Fomentar la pertenencia institucional y personal a las diferentes redes internacionales de intercambio y colaboración.

10. Esforzarse por permeabilizar a los responsables institucionales, sociales y políticos ante las nuevas formas de enseñar y aprender. 


\section{REFERENCIAS}

ADELL, J. (1997). Tendencias en educación en la sociedad de las tecnologías de la información. Edutec, 7, http://ww.ull.es/departamentos/didinv/tecnologia educativa/doc-adell2.html

Bryan, P. (1995). Qué Diccionario para Usuarios de Computadoras. México: Prentice-Hall.

CABERo, J. (2001): Las TICs: una conciencia global en la educación. En CEP de LORCA: Ticemur. Jornadas Nacionales TIC y Educación, Murcia, CEP de Lorca, XIX-XXXVI

COM-EU (2000a). Designing tomorrow's education - Promoting innovation with new technologies. Brussels: Report from the Commission to the Council and the European Parliament EU, http://europa.eu.int/comm/education/elearning/rapen.pdf

COM-EU (2000b). e-Learning - Designing tomorrow's education. Communication from the Commission EU, http://europa.eu.int/comm/education/elearning/comen.pdf

Fahey, T. (1995). Diccionario de Internet. México: Prentice-Hall.

García ARETIO, L. (1985). Licenciados extremeños de la UNED. Badajoz: UNED-Mérida.

García AREtio, L. (1986). Análisis de la eficacia de la educación superior a distancia. Badajoz: UNED-Mérida.

García ARETIO, L. (1987a). Rendimiento académico y abandono en la educación superior a distancia. Madrid: UNED.

GARCía ARETIO, L. (1987b). Eficacia de la UNED en Extremadura. Badajoz: UNED-Mérida.

García Aretio, L. (1989). La educación. Teorías y conceptos. Madrid: Paraninfo.

García Aretio, L. (1992). Las innovaciones y los cambios educativos. En Medina, R., Rodríguez, T. y García Aretio, L. Teoría de la Educación. Madrid: UNED.

García ARETIO, L. (1994). Educación a distancia hoy. Madrid: UNED.

GARCía ARETIO, L. (1999). Historia de la educación a distancia. Revista Iberoamericana de Educación a Distancia (RIED). 2, 1, 11-40.

García Aretio, L. (2001). La educación a distancia; de la teoría a la práctica. Barcelona: Ariel.

García Aretio, L. y Ruiz, M. (2001). Innovación y cambio educativo. En Teoría de la educación. Madrid: UNED, pp. 577-602.

GARRISON, D.R. (2000). Theoretical challenges for distance education in the $21^{\text {st }}$ century: A shift from structural to transactional issues. International Review of Research in Open and Distance Learning, 1, 1.

GisBERT, M. (2000): Las redes telemáticas y la educación del siglo XXI, en Cebrián, M. (coord): Internet en el aula, proyectado el futuro, Málaga, Grupo de Investigación Nuevas Tecnologías Aplicadas a la Educación Universidad de Málaga, 15-25.

Holmberg, B. (1985). Educación a distancia: situación y perspectivas. (traducción de 1981. Londres). Buenos Aires: Kapelusz.

Holmberg, B. (1998). What is new and what is important in distance education. Open Praxis, 1, 32-33.

INGLIS, A. y otros (1999): Delivering digitally, Managing the transition to the knowledge media, London, Kogan Page.

Moore, M.G. (1977). On a theory of independent study. Epistolodidaktika.

Moore, M.G. (1990). Recent contributions to the theory of distance education. Open learning, 5, 3. 
Resistencias, cambio y buenas prácticas en la nueva educación a distancia

ONG, W. (1987). Oralidad y escritura. Tecnologías de la palabra. México: FCE.

OIT (2001). Life at Work in the information economy. World Employment Report 2001. OIT, http://www.oit.org/public/english/support/publ/wer/index2.htm

Perkins, D, (1997). La escuela inteligente. Barcelona: Gedisa.

Peters, O. (1971). Theoretical aspects of correspondence instruction. En McKenzie y Christensen. The changing world of correspondence study. Pensylvania State University Presss.

Peters, O. (1993). Understanding distance education. En Harry, Magnus y Keegan: Distance education: New perspectives. Londres: Routledge.

SALINAS, J. (2000): ¿Qué se entiende por una institución de educación superior flexible?, en CABERO, J. y otros (coods): Y continuamos avanzando. Las nuevas tecnologías para la mejora educativa, Sevilla, Kronos, 451-465.

SEC-EU (2000). A memorandum on lifelong learning. Report European Council at Lisbon, March 2000, http://europa.eu.int/comm/education/life/memoen.pdf

Web-Based Education Commission (2000). The power of the Internet for learning: moving from promise to practice. Moving from promise to practice. Report of the Web-Based Education Comisión. Wa s h i n g t o n , D C, http://www.ed.gov/offices/AC/WBEC/FinalReport/WBECReport.pdf

WedEMEYER, C.A. (1971). Independent study. En Deighton, L.C. (ed) The Encyclopedia of Education, 4. New York: Macmillan.

WEDEMEYER, C. A. (1981). Learning at the back door. Reflections on non-traditional learning in the lifespan. Madison: The University of Wisconsin Press.

WEI YU (2000). Using modern distance education to improve science education in developing countries. World Conference on Science. Sciencefor the twenty-first century. A New Commitment. París: UNESCO.

\section{PERFIL ACADÉMICO Y PROFESIONAL DEL AUTOR}

Lorenzo García Aretio es Titular de la Cátedra UNESCO de Educación a Distancia (www.uned.es/cued). Es doctor y profesor titular de Teoría de la Educación en la Facultad de Educación. Ha sido también Director del Instituto Universitario de Educación a Distancia, Director Adjunto del Programa de Formación del Profesorado y Coordinador general de Planes de Estudio de la UNED. Fue Director-fundador de esta Revista Iberoamericana de Educación a Distancia (RIED) y ahora forma parte de su Comité Científico.

Su línea de investigación se viene centrando desde hace bastantes años en todo lo relativo a la enseñanza/aprendizaje abiertos y a distancia en sus diferentes dimensiones. Como autor principal, coordinador o editor, ha publicado 15 libros relativos al tema de educación a distancia y más de 50 artículos en libros y revistas especializadas. Igualmente viene participando en numerosos proyectos financiados por la Unión Europea, siempre relativos al mismo campo y en los más destacados Congresos internacionales sobre el tema.

Dirección: Lorenzo García Aretio, Facultad de Educación (UNED), Paseo Senda del Rey, 7 - 28040 - Madrid (España) lgretio@edu.uned.es, Tlef.: +34 91 3987218, Fax: +34 913986918 . 